\title{
Antibiotic-associated diarrhoea in emergency department observation unit patients
}

\author{
J. P. HARAN ${ }^{1}$, G. WU ${ }^{1}$, V. BUCCI ${ }^{2}$, A. FISCHER ${ }^{1}$, L. KEANG $^{1}$, E. W. BOYER ${ }^{1}$ \\ AND P. L. HIBBERD ${ }^{3}$ \\ ${ }^{1}$ Department of Emergency Medicine, University of Massachusetts Medical School, Worcester, MA, USA \\ ${ }^{2}$ Department of Biology, University of Massachusetts Dartmouth, North Dartmouth, MA, USA \\ ${ }^{3}$ Department of Pediatrics and Division of Global Health, Harvard Medical School, Boston, MA, USA
}

Received 17 June 2015; Final revision 23 November 2015; Accepted 19 January 2016;

first published online 15 February 2016

\section{SUMMARY}

Clostridium difficile diarrhoea is an urgent threat to patients, but little is known about the role of antibiotic administration that starts in emergency department observation units (EDOUs). We studied risk factors for antibiotic-associated diarrhoea (AAD) and C. difficile infection (CDI) in EDOU patients. This prospective cohort study enrolled adult patients discharged after EDOU antibiotic treatment between January 2013 and 2014. We obtained medical histories, EDOU treatment and occurrence of AAD and CDI over 28 days after discharge. We enrolled and followed 275 patients treated with antibiotics in the EDOU. We found that $52(18.6 \%)$ developed AAD and four (1.5\%) had CDI. Patients treated with vancomycin [relative risk (RR) $0 \cdot 52,95 \%$ confidence interval (CI) 0.3-0.9] were less likely to develop AAD. History of developing diarrhoea with antibiotics (RR 3.11, 95\% CI 1.92-5.03) and currently failing antibiotics (RR 1.90, 95\% CI 1.14-3.16) were also predictors of AAD. Patients with CDI were likely to be treated with clindamycin. In conclusion, AAD occurred in almost $20 \%$ of EDOU patients with risk factors including a previous history of diarrhoea with antibiotics and prior antibiotic therapy, while the risk of $\mathrm{AAD}$ was lower in patients receiving treatment regimens utilizing intravenous vancomycin.

Key words: Antibiotics, Clostridium difficile, diarrhoea, gastrointestinal infections, infectious disease.

\section{INTRODUCTION}

Antibiotic-associated diarrhoea (AAD) is a common complication of antibiotic administration. The frequency of AAD varies among antibacterial agents and is influenced by patient's age and medical comorbidities [1]. Non-white Hispanics have also been shown to have higher rates of AAD [2]. Most studies have evaluated risk factors for AAD in 10-20\% of inpatients that develop the disease [3]. The only emergency

\footnotetext{
* Author for correspondence: J. P. Haran, MD, 55 Lake Avenue North, Worcester, MA 01655, USA.

(Email: john.haran@umassmed.edu)
}

department (ED)-based study found that $18 \%$ of ED patients developed $\mathrm{AAD}-12 \cdot 3 \%$ treated with oral antibiotics alone compared to $25 \cdot 7 \%$ of patients treated with intravenous (IV) antibiotics [2]. Attention continues to focus on AAD being caused by the toxin producing bacteria Clostridium difficile. C. difficile infection (CDI) occurs in 10-20\% of AAD cases [4] and, due to its recent marked increases in both prevalence and severity, has become the leading cause of gastroenterological hospitalizations and deaths accounting for over half a million infections and 29000 deaths annually [5, 6]. Little is known about the role of antibiotics administered in the ED on the development of CDI. 
One-third of US hospitals use emergency department observation units (EDOUs) to treat $>2.3$ million patients presenting to the ED, with the number of patients treated increasing each year [7]. Most EDOUs use specific protocols to treat patients that are referred from the ED for a time period typically $<24$ h [8]. Protocol-driven EDOUs have provided favourable patient outcomes [9], which has allowed the number of disease conditions treated in EDOUs to steadily rise. Although EDOUs are routinely utilized for the treatment of bacterial infections such as skin and soft tissue infections (SSTIs), urinary tract infections, and respiratory tract infections, there is little information on patient outcomes [10,11], particularly occurrence of AAD and CDI.

Over the past decade, failure to treat bacterial infections with outpatient antibiotics has increased by $12 \%$, with more than one in ten first-line antibiotic monotherapies failing [12]. EDOUs are increasingly being used for primary treatment of bacterial infections and after outpatient treatment failure. Little is known about the consequences of the wide range of antibiotics used in EDOUs, particularly AAD and CDI [2]. The objectives of this study were to describe the prevalence of various antibiotics used in EDOUs and the occurrence of any diarrhoeal symptoms, $\mathrm{AAD}$, and CDI over a 1-year period.

\section{METHODS}

\section{Study design}

The University of Massachusetts Medical Center is a 781-bed academic tertiary-care facility that sees $>130000$ emergency visits/year and has a nine-bed 24-h EDOU. This is a prospective cohort of adult EDOU patients who were treated for an acute bacterial infection over a 12-month period from January 2013 to December 2013. The hospital's institutional review board (IRB) approved the study (IRB docket no. H00001871).

\section{Study setting and population}

Patients were eligible for participation if they were discharged home after any duration of stay in the EDOU, and either received antibiotics in the EDOU or upon discharge. Potential subjects were identified using EDOU census logs at the end of each month, and contacted by telephone 28 days after their discharge date and asked if they were willing to participate in a telephone survey. This contact window was selected to span the time during when AAD and CDI symptoms typically occur after taking antibiotics, while trying to minimize recall bias [13]. Patients were eligible if they were English-speaking, aged $\geqslant 18$ years, and had a working phone number. Patients were excluded upon initial phone screening if they had diarrhoea on ED presentation or within the preceding 4 weeks, were unable to cooperate with the questionnaire or recall events surrounding their care, or declined to be interviewed.

\section{Study protocol}

After the patient agreed to participate, a standardized survey was administered by telephone and included questions pertaining to the patient's antibiotic compliance, occurrence of symptoms of AAD or CDI, subsequent healthcare visits/hospitalizations, and any other complication from initial EDOU treatment. Medical histories and allergies were obtained and confirmed through chart review. The Charlson comorbidity index (CCI) was calculated and used to rank patients' medical comorbidities $[14,15]$. Information pertaining to initial ED presentation, EDOU hospital course, and antibiotic treatments used were obtained from the medical records. Symptoms of diarrhoea were selfreported and defined as any loose stools during the observation period. AAD was defined as $\geqslant 3$ loose stools per day for $\geqslant 2$ consecutive days $[3,16]$. Mild diarrhoeal illness was defined as diarrhoeal symptoms not fulfilling the criteria for AAD. CDI was defined as AAD that led to a diagnosis of $C$. difficile infection and/or new treatment prescribed for CDI. $C$. difficile infection was confirmed if there was a report of a stool test positive for $C$. difficile toxins (two of the four CDI patients). Stool samples were tested for the presence and concentration of $C$. difficile toxin A using a 5D8-2C7 mAb pair-based ELISA assay. Study data were collected and managed using REDCap electronic data capture tools hosted at the University of Massachusetts Medical School [17].

\section{Measurements}

The primary study outcomes were the development of any diarrhoeal symptoms, AAD and CDI. Failure of EDOU therapy was defined as incomplete resolution of infectious symptoms that resulted in an additional healthcare visit in which antibiotic therapy was altered either via hospitalization or ambulatory visit. 


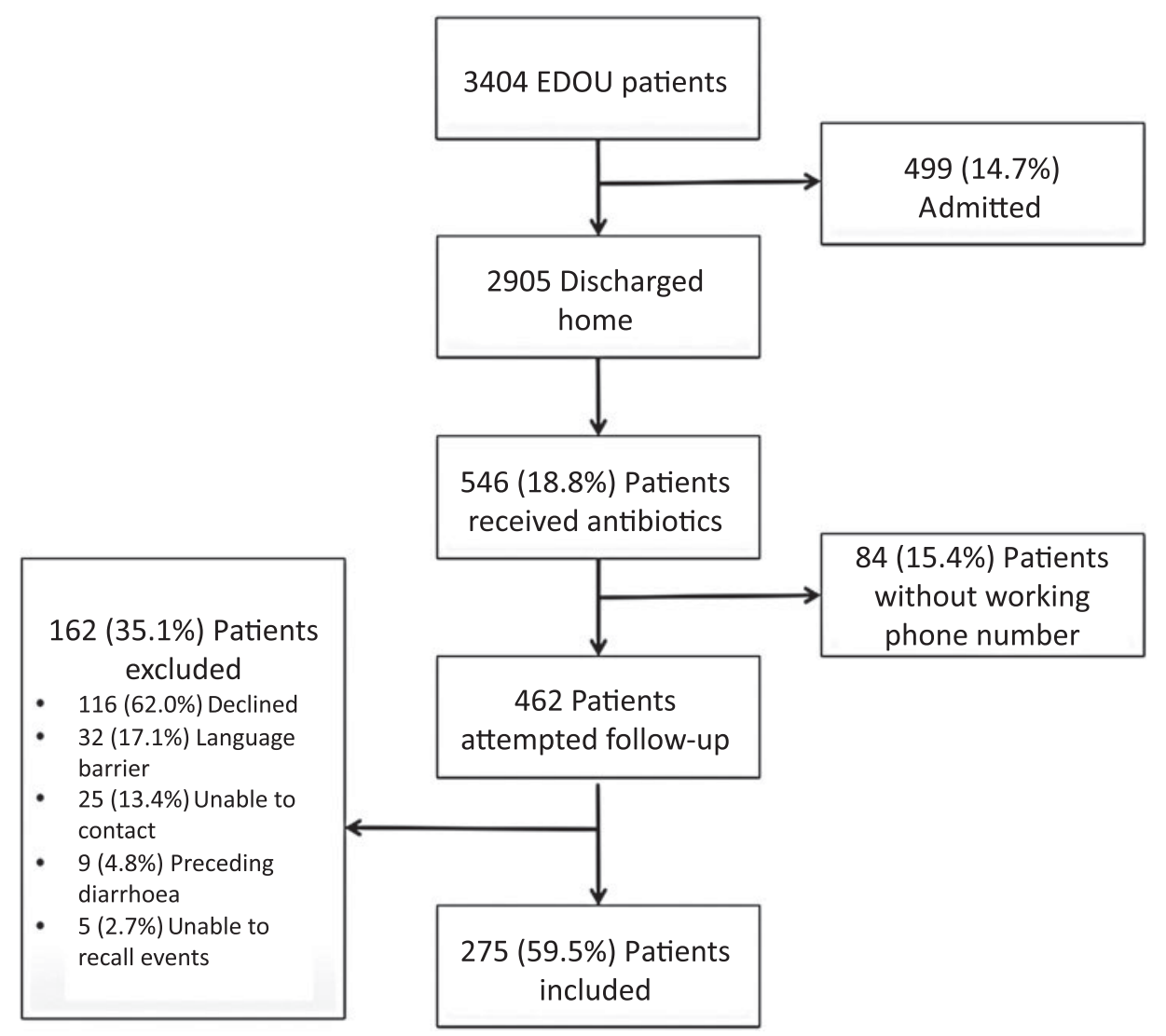

Fig. 1. Flow chart of the study. EDOU, Emergency department observation unit.

\section{Data analysis}

We used $\chi^{2}$ tests to compare rates of AAD in categories of single variables and regression analysis to assess variables' effects, adjusted for the contributions of other variables. The software used for the analysis was Prism Release 6 (GraphPad Software Inc., USA). Regression was performed using the statistical software R (R Studio, USA). We built generalized linear models to perform Poisson regression with robust error variance estimation (PRwREV) for data with binary outcomes [18]. We preferred this approach as it enabled direct estimation of relative risk for a common outcome, in contrast to logistic regression [19]. Due to the large number of covariates, for computational feasibility we focused only on additive effects (i.e. we neglected higher-order interactions). Starting variables for multivariate model building were selected first from an a-priori hypothesis and then added to univariate data analysis. Automated model selection analysis was performed using the glmulti function from the $\mathrm{R}$ package [20]. The optimal model was the one with minimum Akaike's Information Criterion (AIC) [21]. We performed power analysis using simulations [22].
Given the predicted incidence rate of the major statistically significant covariates we generated 10000 random sets of Poisson-distributed binary data. The power of the study to detect different multivariate predictors was determined by simulation, using 10000 random sets of Poisson-distributed binary data. The incidence of AAD was assumed to be $23 \cdot 5 \%$. Based on a sample size of 251 there was $80 \%$ power to detect predictors with a relative risk of $<0.61$ or $>1.39$ and $90 \%$ power to detect relative risk of $<0.53$ or $>1.47$ (two-sided $\alpha 0 \cdot 05$ ). The study did not have sufficient power to reliably detect predictors with relative risks closer to 1 than those specified.

\section{RESULTS}

\section{Characteristics of the study subjects}

During the 1-year study period, there were 2905 patients treated in the EDOU and discharged home (Fig. 1). Of these, 18.8\% were treated with antibiotics for an infection leaving 546 eligible patients. We were unable to contact 84 subjects due to a lack of contact information. Of the remaining 462 patients, 116 
Table 1. Characteristics of study patients

\begin{tabular}{|c|c|c|c|c|c|c|}
\hline \multirow[b]{2}{*}{ Demographics } & \multicolumn{2}{|c|}{ No symptoms } & \multicolumn{2}{|c|}{ Diarrhoea patients } & \multirow[b]{2}{*}{$\mathrm{RR}$} & \multirow[b]{2}{*}{$95 \% \mathrm{CI}$} \\
\hline & $n$ & $(\%)$ & $n$ & $(\%)$ & & \\
\hline Age $\dagger$ & $46 \cdot 7$ & $(17 \cdot 5)$ & $46 \cdot 1$ & $(16 \cdot 9)$ & $1 \cdot 00$ & $(0 \cdot 99-1 \cdot 01)$ \\
\hline Female & 99 & $(49 \cdot 5)$ & 44 & $(58 \cdot 7)$ & $1 \cdot 31$ & $(0 \cdot 88-1 \cdot 94)$ \\
\hline White & 160 & $(80 \cdot 0)$ & 57 & $(76 \cdot 0)$ & $0 \cdot 85$ & $(0 \cdot 54-1 \cdot 31)$ \\
\hline Hispanic & 19 & $(9 \cdot 5)$ & 14 & $(18 \cdot 7)$ & $1 \cdot 68 *$ & $(1 \cdot 07-2 \cdot 65)$ \\
\hline African American & 10 & $(5 \cdot 0)$ & 1 & $(1 \cdot 3)$ & $0 \cdot 32$ & $(0 \cdot 05-2 \cdot 12)$ \\
\hline Asian & 3 & $(1 \cdot 5)$ & 1 & $(1 \cdot 3)$ & $0 \cdot 82$ & $(0 \cdot 17-5 \cdot 06)$ \\
\hline \multicolumn{7}{|l|}{ Medical history } \\
\hline CCI 0 & 125 & $(62 \cdot 5)$ & 49 & $(65 \cdot 3)$ & $1 \cdot 09$ & $(0 \cdot 73-1 \cdot 64)$ \\
\hline CCI 1 & 37 & $(18 \cdot 5)$ & 14 & $(18 \cdot 7)$ & $1 \cdot 01$ & $(0 \cdot 61-1 \cdot 65)$ \\
\hline CCI 2 & 25 & $(12 \cdot 5)$ & 7 & $(9 \cdot 3)$ & $0 \cdot 73$ & $(0 \cdot 39-1 \cdot 56)$ \\
\hline $\mathrm{CCI} \geqslant 3$ & 13 & $(6 \cdot 5)$ & 5 & $(6 \cdot 7)$ & $1 \cdot 02$ & $(0 \cdot 47-2 \cdot 21)$ \\
\hline Hx. diarrhoea & 33 & $(16 \cdot 5)$ & 33 & $(44 \cdot 0)$ & $2 \cdot 49^{*}$ & $(1 \cdot 73-3 \cdot 58)$ \\
\hline Current Abx. & 30 & $(15 \cdot 0)$ & 22 & $(29 \cdot 3)$ & $1 \cdot 78 *$ & $(1 \cdot 20-2 \cdot 64)$ \\
\hline \multicolumn{7}{|l|}{ Condition treated } \\
\hline Cellulitis & 127 & $(63 \cdot 5)$ & 41 & $(54 \cdot 7)$ & $0 \cdot 77$ & $(0 \cdot 52-1 \cdot 13)$ \\
\hline Abscess & 19 & $(9 \cdot 5)$ & 10 & $(13 \cdot 3)$ & $1 \cdot 31$ & $(0 \cdot 76-2 \cdot 25)$ \\
\hline ENT & 20 & $(10 \cdot 0)$ & 9 & $(12 \cdot 0)$ & $1 \cdot 16$ & $(0 \cdot 65-2 \cdot 07)$ \\
\hline UTI & 13 & $(6 \cdot 5)$ & 6 & $(8 \cdot 0)$ & $1 \cdot 17$ & $(0 \cdot 59-2 \cdot 34)$ \\
\hline Pneumonia & 10 & $(5 \cdot 0)$ & 4 & $(5 \cdot 3)$ & $1 \cdot 05$ & $(0 \cdot 45-2 \cdot 46)$ \\
\hline Dental & 8 & $(4 \cdot 0)$ & 3 & $(4 \cdot 0)$ & $1 \cdot 00$ & $(0 \cdot 37-2 \cdot 68)$ \\
\hline Prophylaxis & 3 & $(1 \cdot 5)$ & 2 & $(2 \cdot 7)$ & $1 \cdot 48$ & $(0 \cdot 50-4 \cdot 41)$ \\
\hline Hours in ED $\dagger$ & $20 \cdot 1$ & $(12 \cdot 1)$ & $18 \cdot 8$ & $(12 \cdot 6)$ & $0 \cdot 99$ & $(0 \cdot 98-1 \cdot 01)$ \\
\hline \multicolumn{7}{|l|}{ Treatments } \\
\hline 1st-gen. cephalosporins & 73 & $(36 \cdot 5)$ & 73 & $(37 \cdot 3)$ & $1 \cdot 03$ & $(0 \cdot 69-1 \cdot 53)$ \\
\hline 3rd-gen. cephalosporins & 26 & $(13 \cdot 0)$ & 13 & $(17 \cdot 3)$ & $1 \cdot 27$ & $(0 \cdot 78-2 \cdot 08)$ \\
\hline Vancomycin & 66 & $(33 \cdot 0)$ & 13 & $(17 \cdot 3)$ & $0 \cdot 52 *$ & $(0 \cdot 30-0 \cdot 89)$ \\
\hline Clindamycin & 58 & $(29 \cdot 0)$ & 25 & $(33 \cdot 3)$ & $1 \cdot 16$ & $(0 \cdot 77-1 \cdot 74)$ \\
\hline Macrolide & 12 & $(6 \cdot 0)$ & 5 & $(6 \cdot 7)$ & $1 \cdot 08$ & $(0 \cdot 51-2 \cdot 33)$ \\
\hline Penicillin & 9 & $(4 \cdot 5)$ & 4 & $(5 \cdot 3)$ & $1 \cdot 14$ & $(0 \cdot 49-2 \cdot 63)$ \\
\hline Penicillin/I & 19 & $(9 \cdot 5)$ & 15 & $(20 \cdot 0)$ & $1 \cdot 77 *$ & $(1 \cdot 14-2 \cdot 74)$ \\
\hline Quinolone & 14 & $(7 \cdot 0)$ & 9 & $(12 \cdot 0)$ & $1 \cdot 49$ & $(0 \cdot 86-2 \cdot 59)$ \\
\hline Sulfonamide & 59 & $(29 \cdot 5)$ & 19 & $(25 \cdot 3)$ & $0 \cdot 86$ & $(0 \cdot 55-1 \cdot 34)$ \\
\hline Doxycycline & 21 & $(10 \cdot 5)$ & 3 & $(4 \cdot 0)$ & $0 \cdot 44$ & $(0 \cdot 15-1 \cdot 28)$ \\
\hline Nitrofuantoin & 0 & $(0)$ & 2 & $(2 \cdot 7)$ & $3 \cdot 74 *$ & $(3 \cdot 07-4 \cdot 55)$ \\
\hline Metronidazole & 4 & $(2 \cdot 0)$ & 4 & $(5 \cdot 3)$ & $1 \cdot 88$ & $(0 \cdot 91-3 \cdot 87)$ \\
\hline Number of classes $\dagger$ & $1 \cdot 8$ & $(0 \cdot 9)$ & $1 \cdot 9$ & $(0 \cdot 9)$ & $1 \cdot 06$ & $(0 \cdot 82-1 \cdot 36)$ \\
\hline Abx given intravenously & 147 & $(73 \cdot 5)$ & 55 & $(73 \cdot 3)$ & 0.99 & $(0 \cdot 64-1 \cdot 54)$ \\
\hline Probiotic & 15 & $(7 \cdot 5)$ & 4 & $(5 \cdot 3)$ & $0 \cdot 76$ & $(0 \cdot 31-1 \cdot 85)$ \\
\hline
\end{tabular}

RR, Relative risk; CI, confidence interval; Hx, history; Abx, antibiotics; CCI, Charlson comorbidity index; ENT, ear, nose and throat infection; UTI, urinary tract infection; ED, emergency department; Penicillin/I, penicillin inhibitor combination. $\dagger$ Data represented as means (standard deviation).

$* P<0.05$

declined to be part of the study, 32 could not communicate in English, 25 fell out of the 4-week window for contact, five were unable to recall events surrounding their EDOU stay and nine had diarrhoea within 4 weeks prior to their EDOU admission. The final study consisted of 275 patients. The study population was primarily white non-Hispanic with a mean age of 47 years and a similar distribution of men and women
(Table 1 ). The majority of patients had no medical comorbidities $(\mathrm{CCI}=0)$ with $24 \%$ having previously experienced diarrhoea on antibiotics and $18.9 \%$ of patients currently taking antibiotics prior to the ED visit (i.e. failing outpatient therapy). There were seven categories of infection type treated within the EDOU. The majority of patients were treated for SSTIs (combination of abscess and cellulitis) followed 
by ear, nose and throat infections, urinary tract infections, bacterial pneumonia, dental infections, and finally antibiotic therapy for prophylaxis. The most common antibiotics prescribed were first-generation cephalosporins followed by clindamycin, vancomycin and sulfonamides (i.e. trimethoprim/sulfamethoxazole). Patients on average were treated with more than one class of antibiotic and few patients (6.9\%) were prescribed a probiotic as part of their care.

\section{Main results}

Upon follow-up the majority of patients $(89 \cdot 5 \%)$ completed the full course of antibiotics prescribed to them. Other outcomes included a small percentage of subjects that either failed therapy and required hospitalization $(6.5 \%)$ or had their antibiotics stopped by another provider due to a change in diagnosis of bacterial infection $(5 \cdot 1 \%)$. More than one in four $(27 \cdot 3 \%)$ patients experienced diarrhoea during the course of follow-up with 18.6\% [95\% confidence interval (CI) 13.2-23.2] of patients fulfilling the definition of AAD. Significant differences in patient demographics and treatment regimens were noted between patients that developed diarrhoea and those that did not (Table 1). Hispanics were 68\% more likely to develop diarrhoea on antibiotics than the other racial groups studied [relative risk (RR) $1 \cdot 68,95 \%$ CI $1 \cdot 07-2 \cdot 65$ ]. Additionally patients with a previous history of diarrhoea on antibiotics, and patients presenting to the ED failing antibiotic therapy were more likely to develop diarrhoea than respective comparison groups as a result of their EDOU treatment. Examination of the eight classes of antibiotics given in the EDOU and at discharge, showed that patients that received penicillin inhibitor (penicillin/I) combination antibiotics (i.e. ampicillin/sulbactam) were significantly more likely to develop diarrhoea while patients treated with vancomycin were $48 \%$ less likely to develop diarrhoea (RR $0 \cdot 52,95 \%$ CI $0 \cdot 30-0 \cdot 89$ ).

\section{Effect of multiple antibiotics}

After completion of therapy $44.4 \%$ of patients were treated with only one class of antibiotic, $34 \cdot 5 \%$ received two classes and $21 \cdot 1 \%$ received $\geqslant 3$ classes of antibiotics. We did not observe any differences in the rates of AAD depending on the number of classes used as a whole; however, among specific antibiotic classes there was a significant increase in the rate of AAD when used in combination therapy. Among patients treated with clindamycin, use of clindamycin alone was associated with a markedly reduced risk for developing AAD (14.6\%) compared to patients in whom clindamycin was combined with another class of antibiotics $(31.4 \%, P=0.07)$. Patients receiving clindamycin combination therapy had twice the risk of developing AAD compared to those treated with clindamycin alone (RR $2 \cdot 16,95 \%$ CI $0 \cdot 93-5 \cdot 00$ ). A similar non-significant trend was seen in the penicillin/I combination antibiotic-treated patients $(26 \cdot 7 \%$ vs. $42 \cdot 1 \%, P=0 \cdot 35)$.

\section{Poisson regression model}

To determine the set of covariates significantly affecting the risk of AAD (not any diarrhoea symptoms) we performed PRwREV for data with binary outcome. To determine the model (e.g. set of covariates) best explaining AAD outcome in this population we first built a generalized linear model were AAD was regressed against all the covariates simultaneously. We then used automated-model selection to determine the combination of demographics, medical history and treatment covariates that best fit the AAD profile. The optimal model is:

$$
\begin{aligned}
& \text { AAD } \sim 1+\text { Gender }+ \text { Age }+ \text { HxDiarrhoea } \\
& + \text { CurrentAbx }+ \text { Vancomycin }+ \text { Clindamycin or } \\
& \text { Penicillin or third - generation Cephalosphorin. }
\end{aligned}
$$

Based on the results of this model (Table 2), patients with a history of diarrhoea from previous antibiotic use were three times more likely to develop AAD while patients failing antibiotic therapy (i.e. taking a previously prescribed antibiotic at the time of the ED visit) were $90 \%$ more likely to develop AAD after EDOU discharge. By contrasr, patients treated in the EDOU with vancomycin were $48 \%$ less likely to develop AAD. Patients treated with two of the following three antibiotics: clindamycin, penicillin/I and thirdgeneration cephalosporins were $78 \%$ more likely to go on to develop AAD.

\section{C. difficile cases}

In this study four patients went on to develop CDI (Table 3). These patients were young with no medical comorbidities except one subject with a CCI of 1. Three of the four patients had previously received clindamycin. 
Table 2. Poisson regression model factors affecting the risk of antibiotic-associated diarrhoea

\begin{tabular}{|c|c|c|c|c|c|}
\hline & \multicolumn{2}{|c|}{ Regression coefficient } & \multicolumn{2}{|c|}{ Relative risk } & \multirow[b]{2}{*}{$P$ value } \\
\hline & Average & $95 \% \mathrm{CI}$ & Average & $95 \% \mathrm{CI}$ & \\
\hline Hx. diarrhoea & $1 \cdot 134$ & $(0.651$ to 1.617$)$ & $3 \cdot 109$ & $(1 \cdot 918$ to $5 \cdot 039)$ & $<1 \times 10^{-6}$ \\
\hline Current Abx. & $0 \cdot 641$ & $(0 \cdot 132$ to $1 \cdot 150)$ & $1 \cdot 898$ & $(1 \cdot 141$ to $3 \cdot 157)$ & $0 \cdot 0136$ \\
\hline Vancomycin & -0.661 & $(-1 \cdot 319$ to -0.002$)$ & $0 \cdot 517$ & $(0.267$ to 0.999$)$ & $0 \cdot 0495$ \\
\hline $\begin{array}{l}\text { Clindamycin/penicillin/I/ } \\
\text { 3rd-gen. cephalosporins* }\end{array}$ & $0 \cdot 577$ & $(-0.090$ to $1 \cdot 244)$ & $1 \cdot 781$ & $(0.914$ to 3.471$)$ & $0 \cdot 0897$ \\
\hline
\end{tabular}

CI, Confidence interval; Hx, History; Abx, antibiotics; Penicillin/I, penicillin inhibitor combination.

* Treatment with any two of the above three antibiotics.

Table 3. Characteristics of patients that developed Clostridium difficile infection

\begin{tabular}{lllllllll}
\hline \hline ID & Age & Sex & Race & Hx. diarrhoea & Current Abx. & CCI & Diagnosis & Antibiotics \\
\hline 1 & 31 & F & White & No & Yes & 0 & Abscess & 1st gen., clindamycin \\
2 & 20 & F & Black & No & Yes & 0 & ENT & Clindamycin, macrolide \\
3 & 54 & F & White & No & No & 0 & Cellulitis & Clindamycin \\
4 & 41 & M & Hispanic & Yes & Yes & 1 & ENT & $\begin{array}{c}\text { 3rd gen. cephalosporins, } \\
\text { penicillin/I, macrolide }\end{array}$ \\
\hline \hline
\end{tabular}

Hx, History; Abx, antibiotics; CCI, Charlson comorbidity index; F, female; M, male; Penicillin/I, penicillin inhibitor combination; ENT, Ear, nose and throat infection.

\section{DISCUSSION}

This is the first study to report AAD rates and risk factors for AAD in patients discharged home after a stay in an EDOU. A wide range of antibiotics were used in the EDOU and on discharge from the EDOU. One in four patients treated within the EDOU developed diarrhoea with the majority of these meeting the definition of AAD and $8 \%$ going on to develop CDI. Patients treated with regimens utilizing IV vancomycin were less likely to develop AAD, while patients who had a history of diarrhoea with antibiotics or who had failed outpatient antibiotic therapy were more likely to develop AAD. Combining the use of $\geqslant 2$ antibiotics within the group of antibiotic classes well known for causing AAD (clindamycin, penicillin/I or thirdgeneration cephalosporins) [2] also led to a greatly increased risk of developing AAD.

AAD is a well-known complication of antibiotic therapy [23]. We found similar rates of AAD after a stay in the EDOU as we have previously reported from a multicentre ED cohort study comparing patients treated with IV, but not oral-alone antibiotic regimens [2]. The rate of AAD also falls within the range of other AAD studies involving ambulatory settings [24]. Furthermore, we observed similar antibiotic classes being most frequently associated with AAD, namely clindamycin, third-generation cephalosporins, and penicillin/I antibiotics. When patients received combinations of $\geqslant 2$ of these classes they were $78 \%$ more likely to develop AAD compared to all other treatment regimens after controlling for confounders. Clindamycin was associated with the majority of CDI cases. Interestingly, we found that patients receiving initial ED antibiotic therapy using IV vancomycin were $48 \%$ less likely to develop diarrhoea compared to patients who were not treated with vancomycin. Pharmacologically, this finding makes sense since intravenously delivered vancomycin has poor penetration through the intestinal wall [25]. Vancomycin may be a better first-line IV therapy especially for SSTIs if, through its use, patients have a reduced risk for AAD and CDI.

Other significant factors associated with the development of AAD were if the patient had a history of diarrhoea on antibiotics or if they presented to the ED currently taking antibiotics failing outpatient therapy. The effect of antibiotics on the gut microbiome are profound with a rapid loss of diversity that often does not return fully to its initial state [26]. A history of AAD 
may lead to an increased risk of further developing AAD and CDI due to persistent changes in the gut microbiome [27]. Additionally, if significant changes in the microbiome commence $24 \mathrm{~h}$ after initiating antibiotics [28], introducing an antibiotic change, especially into a broad spectrum antibiotic class, may be too much for the native gut flora to handle thus leading to AAD. The higher rate of AAD in Hispanic patients in this study confirms our previous finding reported from within the general ED population [2]. Further investigation into how the Hispanic gut flora responds to an antibiotic challenge may provide insight into this clinical observation.

A limitation of this study is that it reports data from a single site. This study is also limited in the number of patients enrolled and cases recorded. Following up this investigation with a multi-centre cohort study would strengthen the findings; however, we are reporting on similar trends already reported from our previous larger multi-centre ED investigation. Of the potential patient population we were only able to enrol $60 \%$ of patients approached which represents a potential source of bias; however, these enrolment success numbers are consistent with other observational studies.

In conclusion, based on our findings, we recommend that there is an urgent need to develop antibiotic guidelines to reduce the healthcare burden of AAD during and after admission to EDOUs and to consider adjuvant treatment, such as probiotics, for those at highest risk of developing AAD. Given that EDOUs treat $>2.3$ million patients annually in the United States alone [7], antibiotic guidelines and preventative treatments could affect 34000 cases of CDI annually. Vancomycin as an initial antibiotic treatment may be considered when administering IV therapy for a SSTI where MRSA coverage is warranted; however, its relation to lower risks for AAD needs further study.

\section{DECLARATION OF INTEREST}

None.

\section{REFERENCES}

1. McFarland LV. Epidemiology, risk factors and treatments for antibiotic-associated diarrhea. Digestive Diseases 1998; 16: 292-307.

2. Haran JP, et al. Factors influencing the development of antibiotic associated diarrhea in ED patients discharged home: risk of administering IV antibiotics. American Journal of Emergency Medicine 2014; 32: 1195-1199.
3. Wistrom J, et al. Frequency of antibiotic-associated diarrhoea in 2462 antibiotic-treated hospitalized patients a prospective study. Journal of Antimicrobial Chemotherapy 2001; 47: 43-50.

4. Bartlett JG. Clinical practice. Antibiotic-associated diarrhea. New England Journal of Medicine 2002; 346: 334-339.

5. Hall AJ, et al. The roles of Clostridium difficile and norovirus among gastroenteritis-associated deaths in the United States, 1999-2007. Clinical Infectious Diseases 2012; 55: 216-223.

6. Ghose C. Clostridium difficile infection in the twenty-first century. Emerging Microbes \& Infections 2013; 2: e62.

7. Venkatesh AK, et al. Use of observation care in US emergency departments, 2001 to 2008. PLoS ONE 2011; 6: e24326.

8. Mace SE, et al. A national survey of observation units in the United States. American Journal of Emergency Medicine 2003; 21(7).

9. Ross MA, et al. Protocol-driven emergency department observation units offer savings, shorter stays, and reduced admissions. Health Affairs 2013; 32: 2149-2156.

10. Moseley MG, et al. Emergency department observation units and the older patient. Clinics in Geriatric Medicine 2013; 29: 71-89.

11. Ross MA, et al. The use and effectiveness of an emergency department observation unit for elderly patients. Annals of Emergency Medicine 2003; 41: 668-677.

12. Currie CJ, et al. Antibiotic treatment failure in four common infections in UK primary care 1991-2012: longitudinal analysis. British Medical Journal 2014; 349: g5493.

13. Hickson M. Probiotics in the prevention of antibioticassociated diarrhoea and Clostridium difficile infection. Therapeutic Advances in Gastroenterology 2011; 4: 185-197.

14. Charlson ME, et al. A new method of classifying prognostic comorbidities in longitudinal studies: development and validation. Journal of Chronic Diseases 1987; 40: 373-383.

15. Quan H, et al. Updating and validating the Charlson comorbidity index and score for risk adjustment in hospital discharge abstracts using data from 6 countries. American Journal of Epidemiology 2011; 173: 676-682.

16. McFarland LV, et al. Nosocomial acquisition of Clostridium difficile infection. New England Journal of Medicine 1989; 320: 204-210.

17. Harris PA, et al. Research electronic data capture (REDCap) - a metadata-driven methodology and workflow process for providing translational research informatics support. Journal of Biomedical Sciences 2009; 42: 377-381.

18. Zou G. A Modified Poisson regression approach to prospective studies with binary data. American Journal of Epidemiology 2004; 159: 702-706.

19. Chen W, et al. Comparison of robustness to outliers between robust poisson models and log-binomial models when estimating relative risks for common binary outcomes: a simulation study. BMC Medical Research Methodology 2014; 14: 1-8. 
20. Calcagno V, et al. glmulti: an R package for easy automated model selection with (generalized) linear models. Journal of Statistical Software 2010; 34: 1-29.

21. Brooks DG. Akaike Information Criterion Statistics. Technometrics 1989; 31: 270-271.

22. Landau S, et al. Sample size and power calculations for medical studies by simulation when closed form expressions are not available. Statistical Methods in Medical Research 2013; 22: 324-345.

23. Bartlett JG. Clinical practice. Antibiotic-associated diarrhea. New England Journal of Medicine 2002; 31: 334-339.

24. McFarland LV. Meta-analysis of probiotics for the prevention of antibiotic associated diarrhea and the treatment of Clostridium difficile disease. American Journal of Gastroenterology 2006; 101: 812-822.
25. Rao S, et al. Systemic absorption of oral vancomycin in patients with Clostridium difficile infection. Scandinavian Journal of Infectious Diseases 2011; 43: 386-388.

26. Dethlefsen L, et al. Incomplete recovery and individualized responses of the human distal gut microbiota to repeated antibiotic perturbation. Proceedings of the National Academy of Sciences USA 2011; 108 (Suppl. 1): $4554-4561$.

27. Buffie CG, et al. Precision microbiome reconstitution restores bile acid mediated resistance to Clostridium difficile. Nature 2015; 517: 205-208.

28. De La Cochetiere MF, et al. Resilience of the dominant human fecal microbiota upon short-course antibiotic challenge. Journal of Clinical Microbiology 2005; 43: 5588-5592. 